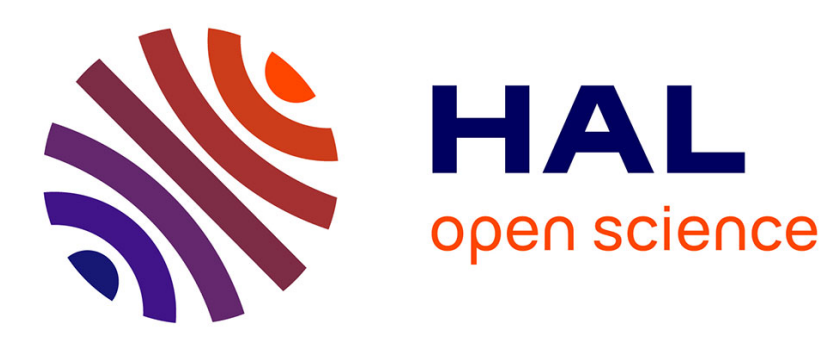

\title{
Fast chaotic optimization algorithm based on locally averaged strategy and multifold chaotic attractor
}

\author{
Tayeb Hamaizia, René Lozi
}

\section{To cite this version:}

Tayeb Hamaizia, René Lozi. Fast chaotic optimization algorithm based on locally averaged strategy and multifold chaotic attractor. 2011. hal-00606509v2

\section{HAL Id: hal-00606509 \\ https://hal.science/hal-00606509v2}

Preprint submitted on 16 Jul 2011

HAL is a multi-disciplinary open access archive for the deposit and dissemination of scientific research documents, whether they are published or not. The documents may come from teaching and research institutions in France or abroad, or from public or private research centers.
L'archive ouverte pluridisciplinaire HAL, est destinée au dépôt et à la diffusion de documents scientifiques de niveau recherche, publiés ou non, émanant des établissements d'enseignement et de recherche français ou étrangers, des laboratoires publics ou privés. 


\title{
Fast chaotic optimization algorithm based on locally averaged strategy and multifold chaotic attractor
}

\author{
Tayeb Hamaizia · René Lozi
}

Received: date / Accepted: date

\begin{abstract}
Recently, chaos theory has been used in the development of novel techniques for global optimization , and particularly, in the specification of chaos optimization algorithms (COA) based on the use of numerical sequences generated by means of chaotic map.

In this paper, we present an improved chaotic optimization algorithm using a new two-dimensional discrete multifold mapping for optimizing nonlinear functions(ICOMM). The proposed method is a powerful optimization technique, which is demonstrated when three nonlinear functions of reference are minimized using the proposed technique.
\end{abstract}

Keywords Chaos optimization algorithms - Nonlinear test functions $\cdot 2$-D Discrete map $\cdot$ multifold chaotic attractor.

\section{Introduction}

Application of chaos in industrial and applied problems is a very important and urgent research topic [1-5] . In particulary in theory of control, cryptography and more recently in global optimization algorithms where introduction of chaotic numbers instead of random ones leads to better results [6]. In general, chaos has three main dynamics properties [7]: sensitive dependence on initial conditions assessed by Lyapunov exponents [8-10], stochasticity and ergodicity.

Taking advantage of properties as ergodicity and stochasticity of chaos, some new algorithms called chaos optimization

\section{Tayeb Hamaizia}

Department of Mathematics, University of Constantine, (25000),Algeria.

Tel.:+213-31-818987

Fax.:+213-31-818987

E-mail: el.tayyeb@umc.edu.dz

René Lozi

Laboratoire J.A. Dieudonné, UMR CNRS 6621 Université de Nice Sophia-Antipolis, Parc Valrose 06108 NICE Cedex 02, France.

E-mail: rlozi@unice.fr algorithm (COA) and hybridization with other techniques are presented in the literature: gradient-based methods [11], genetic algorithms [12, 13], particle swarm optimization [1418], differential evolution [19, 20], clonal algorithms [21], artificial immune systems [22, 23], bee colony algorithms [24] and simulated annealing [25].

The aim of this paper is to present a new optimization algorithm chaotic based on new 2-D discrete chaotic system (map) with multifold attractor.

The paper is organized as follows: in Sec. 2 we present a new strategy based on locally averaged strategy of the global search and multifold chaotic attractor, in Sec. 3 we analyse the effectiveness of the proposed algorithm on a benchmark suite of 3 well-known nonlinear test functions which are optimized. Finally, we propose a conclusion.

\section{Chaotic optimization method}

\subsection{Multifold chaotic attractor}

Since the pioneer chaotic map introduced by Hénon [26] in 1976, many other chaotic maps have been studied [27-29]. Among these maps, some display multifold patterns [30, 31]. In this paper we have used the map recently introduced by Zeraoulia and Sprott, as a modification of Hénon map.

$$
\left\{\begin{array}{l}
y_{1}(k)=1-a\left(\sin _{1}(k-1)\right)+b y(k-1) \\
y(k)=y_{1}(k-1)
\end{array}\right.
$$

where $k$ is the iteration number.

The essential motivation to replace the quadratic term $x^{2}$ in the Hénon map by the nonlinear term in $\sin x$. is to develop a $C^{\infty}$ mapping that is capable of generating chaotic attractors with multifolds via a period-doubling bifurcation route to chaos which has not been studied before in the literature. 
The fact that this map is $C^{\infty}$ in some ways simplifies the study of the map and avoids some problems related to the lack of continuity or differentiability of the map. The choice of the term $\sin x$ has an important role in that it makes the solutions bounded for values of $b$ such that $|b| \leq 1$, and all values of $a$, while they are unbounded for $|b|>1$. The chosen parameters values are $a=4$ and $b=0.9$ as suggested in [30]. For this values the observed attractor (see Fig.1) be-

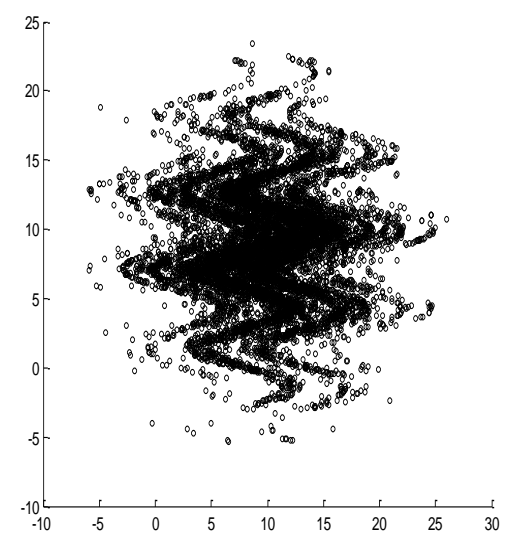

Fig. 1 Chaotic multifold attractor of the map (1) obtained for $a=4$ and $b=0.9$.

longs to the square $\left(y_{1}, y\right) \in[-8.588,27.645]^{2}=D \in R^{2}$. However, even if for all $a$ in $R$ and $|b|<1$ and all initial conditions $\left(y(0), y_{1}(0)\right)$, the orbits of $(1)$ are bounded (see cite26, theorem 5), this map exhibits very complicated dynamical behaviors with coexisting attractors. Hence in order to choose initial conditions for ICOMM for which the attractor is observed we choose it in a subset of $D$. The optimization algorithm needs to normalize the variable $y(k)$ in the range $[0,1]$ using the transformation

$z(k)=\frac{y(k)-\alpha}{\beta-\alpha}$.

where $[\alpha, \beta]=[-8.588,27.645]$

Numerical computation leads to the density $d(s)$ of iterated values of $y(k)$ displayed on Fig. 2. In this figure, the density is normalized to 1 over the whole interval $[0,1]$ i.e.

$$
\int_{0}^{1} d(s) d s=1
$$

Remark: Contrary to the theorical proof that Lozi map exhibits a strange chaotic attractor [32] there is only weak numerical evidence that (1) has chaotic attractors. It is possible that Fig. 1 displays only a transient regime wich leads eventually to a periodic orbit. Numerically, with any initial condition in the basin of attraction defined for $a=4$ and $b=0.9$ one finds a period 6 attractor when $k \geq 150,000$

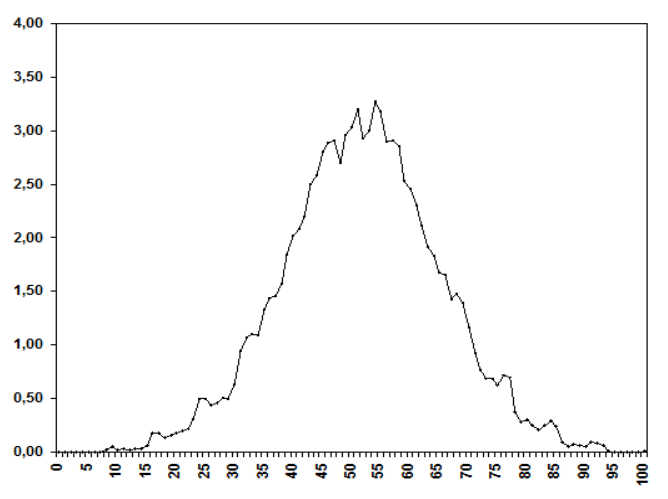

Fig. 2 density of iterated values of $y(k)$ of equation (1) over the interval $[0,1]$ splitted in 100 boxes for 100,000 iterated values.

$$
\begin{aligned}
& y_{1}(k+6)=y_{1}(k)=10.9694028942956052 . \\
& y_{1}(k+1)=13.0613259136267086 \\
& y_{1}(k+2)=8.97249334266406606 \\
& y_{1}(k+3)=11.0071070225514713 \\
& y_{1}(k+4)=13.0749780033934186 \\
& y_{1}(k+5)=8.95855079898761808
\end{aligned}
$$

computation being done with double precision numbers. Again there is no proof that (1) possesses orbit shifted shadowing property as proved for generalized Lozi map [33]. However, as for optimization algorithm only few iterates (i.e. $k \leq 10,000$ ) are needed, property of transient regime ( which is ergodic and stochastic within its range of value) worthes for ICOMM.

\subsection{Locally averaged strategy}

COMM is mainly COLM ( chaotic optimization method based on Lozi map) defined by Coelho [34] in which Lozi map is replaced by the map of Zeraoulia and Sprott. In order to improve COLM we have done [35] some modification in the global step of reaserch. This new algorithm is called ICOLM ( Improved COLM). Now we introduce the modification to COMM in order the global search converges. The ICOMM algorithm is then defined as:

$$
\begin{gathered}
\text { Find } X \text { to minimize } f(X), X=\left[x_{1}, x_{2}, x_{n}\right] \\
\text { subject to } x_{i} \in\left[L_{i}, U_{i}\right] .
\end{gathered}
$$

Where $f$ is the objective function, and $X$ is the decision solution vector consisting of $n$ variables $x_{i} \in R^{n}$ bounded by lower $\left(L_{i}\right)$ and upper limits $\left(U_{i}\right)$. The chaotic search procedure based on two-dimensional maps can be illustrated as 
follows [34, 36, 37]:

Inputs:

$M_{g}$ : max number of iterations of chaotic Global search.

$\mathrm{Mgl}_{1}$ : max number of iterations of first chaotic Local search in Global search.

$\mathrm{Mgl}_{2}$ : max number of iterations of second chaotic Local search in Global search.

$M_{l}$ : max number of iterations of chaotic Local search.

$M t=M g \times\left(M g l_{1}+M g l_{2}\right)+M g$ : stopping criterion of chaotic optimization method in iterations.

$\lambda_{g l 1}$ : step size in first global-local search.

$\lambda_{g l 2}$ : step size in second global-local search.

$\lambda$ : step size in chaotic local search.

Outputs:

$\bar{X}$ : best solution from current run of chaotic search.

$\bar{f}$ : best objective function (minimization problem).

-Step 1 : Initialization of the numbers $M_{g}, M_{g l 1}, M_{g l 2}$, $M_{l}$ of steps of chaotic search and initialization of parameters $\lambda_{g l 1}, \lambda_{g l 2}, \lambda$ and initial conditions. Set $k=1, y(0), y_{1}(0)$, $a=4$ and $b=0.9$. Set the initial best objective function $\bar{f}=+\infty$

- Step 2: algorithm of chaotic global search:

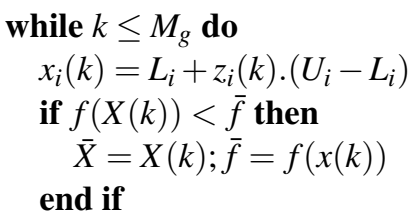

- Step 2-1: sub algorithm of first chaotic global-local search:

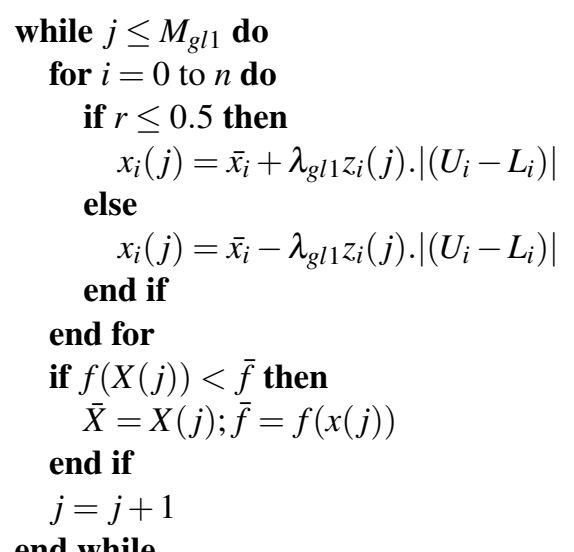

end while

- Step 2-2: sub algorithm of second chaotic globallocal search:

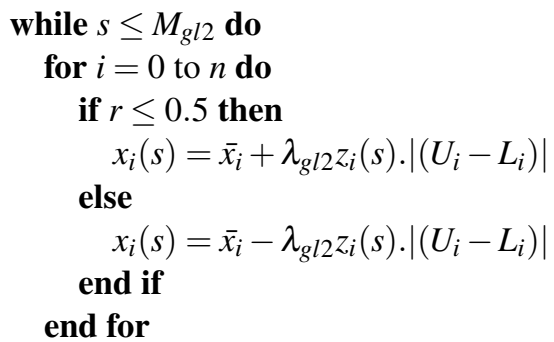

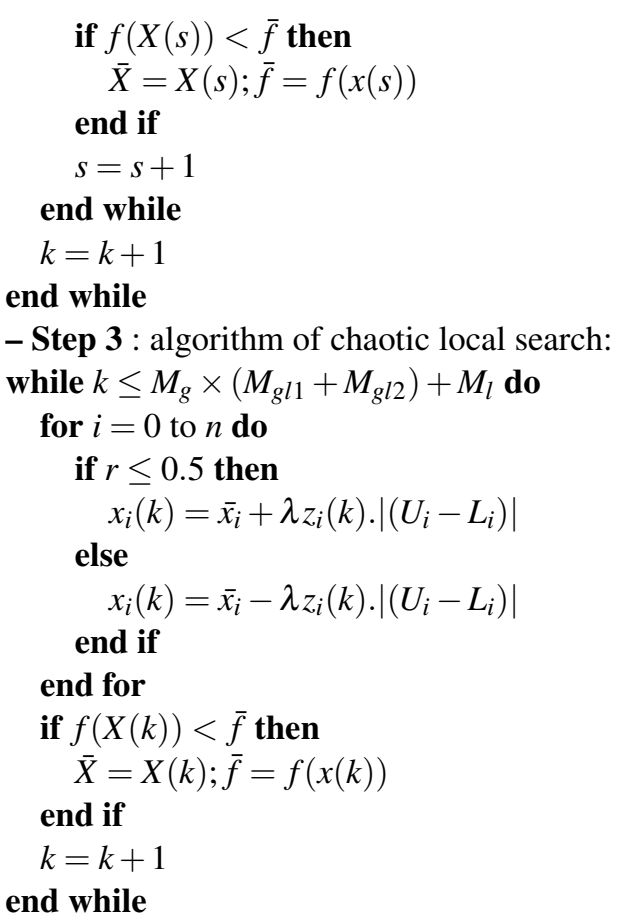

Heuristics: the locally averaged strategy of ICOMM and ICOLM leads to better results than COMM or COLM as shown on Fig.3. In this figure only three global search results are displayed $x_{1}, x_{2}, x_{3}$ with

$f\left(x_{2}\right)<f\left(x_{3}\right)<f\left(x_{1}\right)$.

The local search following global one starts from the best global result $x_{2}$ (from (3)) and gives $x_{2}^{\prime}$. Instead the local-global search around $x_{1}, x_{2}$ and $x_{3}$, leads to $x_{1}^{\prime}, x_{2}^{\prime}, x_{3}^{\prime}$ which verify

$f\left(x_{1}^{\prime}\right)<f\left(x_{3}^{\prime}\right)<f\left(x_{2}^{\prime}\right)$.

The local search following the local-global one starts now from the best globally averaged result $x_{1}^{\prime}(\operatorname{from}(4))$ and leads to $x_{F}$

$f\left(x_{F}\right)<\left(x_{1}^{\prime}\right)$.

\section{Experiments and analysis}

In this section, the benchmark suite consists in three nonlinear multimodal functions that differ in terms of various characteristics. They are used to evaluate application performance of ICOMM. To examine the effectiveness of this method involving the multifold map, we apply ICOMM for each function. We use different values of steps size $\lambda, \lambda_{g l 1}$ and $\lambda_{g l 2}$. For each trial we use 48 random initial points ( 48 runs); on a $3.2 \mathrm{GHz}$ Pentium IV processor with $2 \mathrm{~GB}$ of RAM. For all the studied cases, the four configurations, numbered from $\mathrm{C} 1$ to $\mathrm{C} 4$, that are used are presented in Tab. 1 


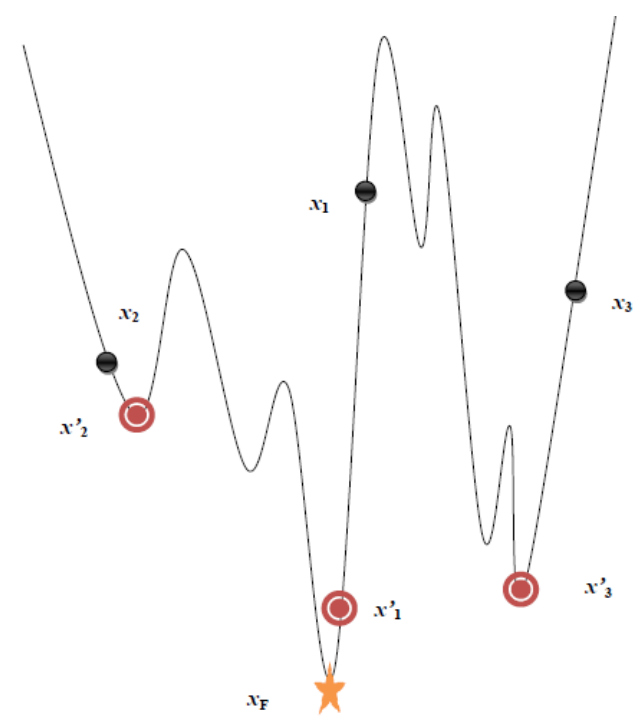

Fig. 3 Heuristics of locally-averaged strategy.

\begin{tabular}{|l|l|l|l|l|l|l|l|l|}
\cline { 2 - 9 } \multicolumn{1}{c|}{} & $\lambda$ & $\lambda_{M_{g l 1}}$ & $\lambda_{M_{g l 2}}$ & $M_{g}$ & $M_{l}$ & $M_{g l 1}$ & $M_{g l 2}$ & $M t$ \\
\hline $\mathrm{C} 1$ & 0.01 & 0.04 & 0.01 & 10 & 50 & 2 & 2 & 90 \\
\hline $\mathrm{C} 2$ & 0.01 & 0.4 & 0.01 & 10 & 50 & 2 & 2 & 90 \\
\hline C4 & 0.01 & 0.04 & 0.01 & 100 & 50 & 5 & 5 & 1050 \\
\hline
\end{tabular}

Table 1 The set of parameters values for every run on the benchmark suite defined in Sec. 2.2

\subsection{Multimodal test functions}

We test ICOMM in 2D optimization problem using :

\subsubsection{Function $f_{1}$ (See Fig. 4)}

The function $f_{1}$ is the Easom function $[2,5]$

$$
f_{1}=-\cos \left(x_{1}\right) \cos \left(x_{2}\right) e^{\left.\left(-\left(x_{1}-\pi\right)^{2}-\left(x_{2}-\pi\right)^{2}\right)\right)}
$$

its characteristics are:

- search domain:-10 $\leq x_{i} \leq 10, i=1,2$.

- number of local minima: several local minima.

- one global minimum: $\bar{x}=(\pi, \pi), f(\bar{x})=-1$.

\subsubsection{Function $f_{2}$ (See Fig. 5)}

The function $f_{2}$ is the Rosenbrock's function $[2,5]$

$$
f_{2}=100\left(x_{1}^{2}+x_{2}\right)^{2}+\left(1-x_{1}\right)^{2}
$$

its characteristics are defined as follows:

- search domain: $-2.048 \leq x_{i} \leq 2.048, i=1,2$.

- number of local minima: several local minima.

- The global minimum: $\bar{x}=(0,0), f(\bar{x})=0$.

\subsubsection{Function $f_{3}$ (See Fig. 6)}

The function $f_{3}$ is more complex $[35,38]$ than $f_{1}$ and $f_{2}$

$$
\begin{gathered}
f_{3}=x_{1}^{4}-7 x_{1}^{2}-3 x_{1}+x_{2}^{4}-9 x_{2}^{2}-5 x_{2} \\
+11 x_{1}^{2} x_{2}^{2}+99 \sin \left(71 x_{1}\right)+137 \sin \left(97 x_{1} x_{2}\right)+131 \sin \left(51 x_{2}\right) \\
\text { - search domain: }-10 \leq x_{i} \leq 10, i=1,2 . \\
\text { - number of local minima: several local minima. }
\end{gathered}
$$

The essential feature of this benchmark function is that location of minima is not symmetric. In a forthcoming paper we will extend our numerical analysis in higher dimension with an extended benchmark suite [38].

\subsection{Numerical results}

The numerical results are displayed in Tab.2.

For both functions $f_{1}$ and $f_{2}$ the global minimum is easily reached in few steps. Configurations $\mathrm{C} 1$ and $\mathrm{C} 2$ are fast and efficient. Concerning $f_{3}$ which possesses hundreds of local minima, the best results are obtained using configurations C3 and C4. The global minima is not yet theoretically known, however extended numerical computations give some clues that the values of $f_{3}$ found using both $\mathrm{C} 3$ and $\mathrm{C} 4$ are not far from the value of $f_{3}$ on the global minimum. The locally averaged strategy of ICOMM is illustrated on Fig.7 on which the result of every step 2-2 is plotted.

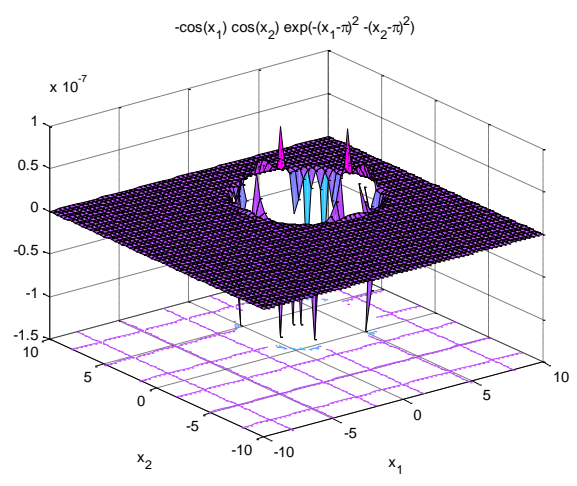

Fig. 4 graph of test function $f_{1}$ in the search domain 


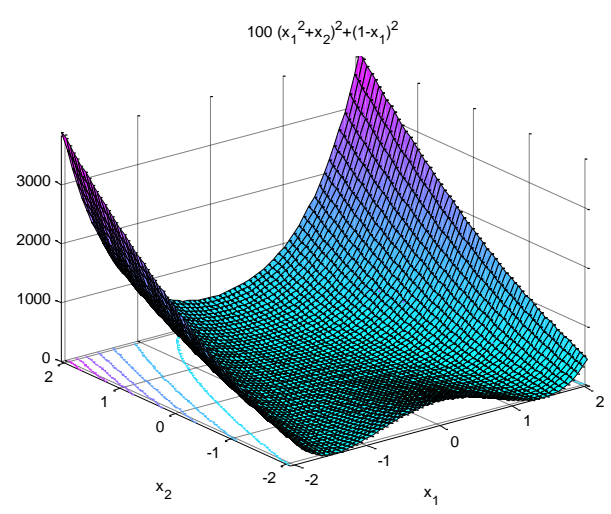

Fig. 5 graph of test function $f_{2}$ in the search domain

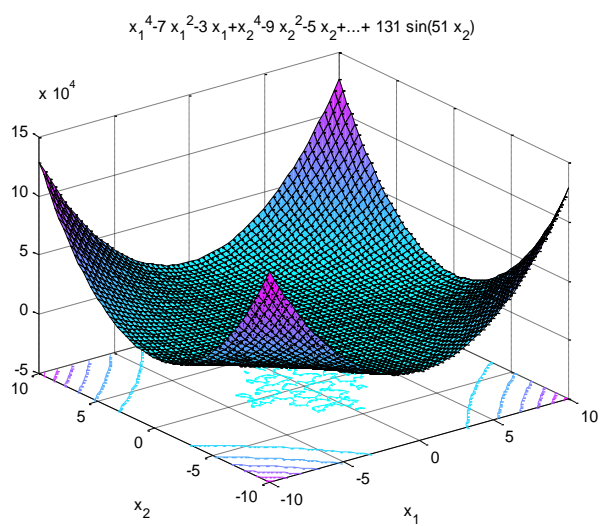

Fig. 6 graph of test function $f_{3}$ in the search domain

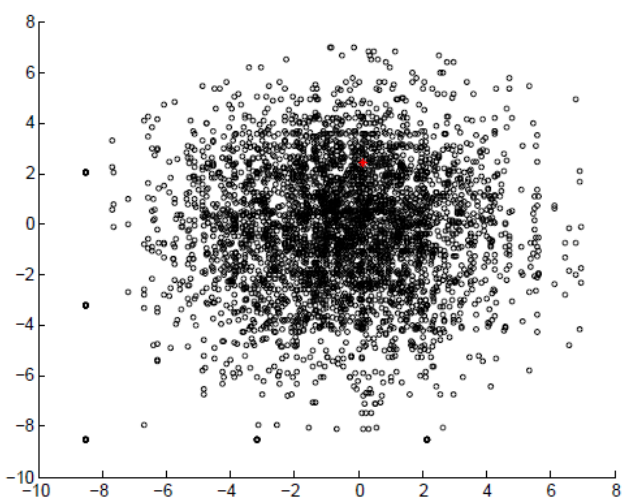

Fig. 7 Locally-averaged strategy of chaotic search. Results of every Step 2-2 for $f_{3}$

\section{Conclusion}

In this paper, we have presented a new chaotic optimization algorithm inspired by COLM methods, chaos optimization algorithms based on new 2-D discrete multifold chaotic attractor. This algorithm is tested on a benchmark suite consisting in three well know nonlinear reference functions.

The presented study allows us to conclude that the proposed

\begin{tabular}{|l|l|l|l|l|l|}
\cline { 2 - 6 } \multicolumn{1}{c|}{} & Best value & $\begin{array}{l}\text { Mean } \\
\text { value }\end{array}$ & Std.Dev & $(\mathrm{x}, \mathrm{y})$ & $\mathrm{T} / \mathrm{s}$ \\
\hline$f_{1}$ & -1.0000 & -0.9999 & 0.0001 & $(3.1443,3.1443)$ & 1.9490 \\
& -1.0000 & -0.9998 & 0.0002 & $(3.1458,3.1446)$ & 1.9499 \\
& -1.0000 & -0.9999 & 0.0001 & $(3.1453,3.1445)$ & 27.8084 \\
& -1.0000 & -1.0000 & 0.0000 & $(3.1420,3.1420)$ & 55.5564 \\
\hline$f_{2}$ & 0.0000 & 0.0000 & 0.0000 & $(0.9996,0.9978)$ & 1.8380 \\
& 0.0000 & 0.0000 & 0.0000 & $(0.9988,0.9977)$ & 1.8386 \\
& 0.0000 & 0.0000 & 0.0000 & $(0.9999,0.9998)$ & 25.9905 \\
& 0.0000 & 0.0000 & 0.0000 & $(1.0001,1.0002)$ & 52.1532 \\
\hline$f_{3}$ & -373.2600 & -362.8730 & 5.8505 & $(-0.2926,-2.6142)$ & 2.1350 \\
& -391.1240 & -362.9798 & 10.7292 & $(-2.0556,-2.4995)$ & 2.1736 \\
& -395.5435 & -390.5618 & 5.1234 & $(-0.2897,-0.2786)$ & 31.3117 \\
& -395.5870 & -391.3068 & 4.7932 & $(-0.2034,0.0920)$ & 62.2426 \\
\hline
\end{tabular}

Table 2 optimization results over 48 runs for 4 parameter configurations

method is fast and converges to a good optimum. because we used a sampling mechanism to coordinate the research methods based on chaos theory, and we refined the final solution using a second method of local search. Further research is needed to gain more confidence and better understanding of the proposed methodology. The proposed algorithm has to be evaluated for a large number of test functions in higher dimension.

\section{References}

1. Li, B., Jiang, W.S. :Chaos optimization method and its application. Journal of Control Theory and Application. 14(4), 613-615 (1997)

2. Li, B., Jiang, W.S. :Optimizing complex function by chaos search. Cybernetics and Systems, 29(4), 409-419 (1998)

3. Choi, C., Lee, J.J. :Chaotic local search algorithm. Artificial Life and Robotics, 2(1), 41-47 (1998)

4. Zhang, C., Xu, L., Shao, H. :Improved chaos optimization algorithm and its application in nonlinear constraint optimization problems. Shanghai Jiaotong Daxue Xuebao, Journal of Shanghai Jiaotong University, 34(5), 593-595 (2000)

5. Yang, D., Li, G., Cheng,G. : On the efficiency of chaos optimization algorithms for global optimization. Chaos, Solitons and Fractals, 34, 1366-1375 (2007)

6. Liu,Y., Tang,W.K.S.: Modified dynamic minimization algorithm for parameter estimation of chaotic system from a time series. Nonlinear Dyn. DOI 10.1007/s11071-010-9922-0 (2011)

7. Tavazoei, M. S., Haeri, M. :An optimization algorithm based on chaotic behavior and fractal nature. Journal of Computational and Applied Mathematics. 206(2), 1070-1081 (2007)

8. Strogatz, S.H.: Nonlinear Dynamics and Chaos. Massachussetts: Perseus Publishing, (2000) 
9. Caponetto, R., Fortuna, L., Fazzino, S., Xibilia, M.G. :Chaotic sequences to improve the performance of evolutionary algorithms. IEEE Transactions on Evolutionary Computation. 7(3) 289-304 (2003)

10. Wu, X.X., Chen, Z.: Introduction of Chaos Theory, Shanghai Science and Technology, Bibliographic Publishing House. (1996).

11. Ou-Yang, Y.X., Tang, M., Liu, S.L., Dong, J.X. : Combined BFGS-chaos method for solving geometric constraint. Zhejiang Daxue Xuebao (Gongxue Ban)/Journal of Zhejiang University (Engineering Science). 39(9), 1334-1338 (2005)

12. Fei, C.G., Han, Z.Z.: A novel chaotic optimization algorithm and its applications. Journal of Harbin Institute of Technolog y (New Series). 17(2),254-258 (2010)

13. Tenreiro Machado,J.A.:Optimal tuning of fractional controllers using genetic algorithms. Nonlinear Dyn. 62, 447-452 (2010)

14. Liu, B., Wang, L., Jin, Y.H., Tang, F., Huang, D.X. :Improved particle swarm optimization combined with chaos. Chaos, Solitons and Fractals. 25(5),1261-1271 (2005)

15. Chen, R.Q., Yu, J.S.: Study and application of chaosparticle swarm optimization-based hybrid optimization algorithm. Xitong Fangzhen Xuebao / Journal of System Simulation. 20(3), 685-688 (2008)

16. Meng, H.J, Zheng, P., Wu, R.Y., Hao, X.-J., Xie,Z.: A hybrid particle swarm algorithm with embedded chaotic search. 2004 IEEE Conference on Cybernetics and Intelligent Systems, 367-371 (2004)

17. Alatas, B., Akin, E., Ozer, A. B.: Chaos embedded particle swarm optimization algorithms. Chaos, Solitons and Fractals, 40(4), 1715-1734 (2009)

18. Wei,Z., Ge,F., Lu, Y., Li,L., Yang,Y.:Chaotic ant swarm for the traveling salesman problem. Nonlinear Dyn. DOI 10.1007/s11071-010-9889-x (2010)

19. Guo, Z.Y., Kang, L.Y., Cheng, B.,Ye, M., Cao, B.G. : Chaos differential evolution algorithm with dynamically changing weighting factor and crossover factor. Harbin Gongcheng Daxue Xuebao/Journal of Harbin Engineering University. 27, 523-526 (2006)

20. Ho, W.-H., Chou, J.-H., Guo, C.-Y.: Parameter identification of chaotic systems using improved differential evolution algorithm..Nonlinear Dyn.Nonlinear Dyn. 61, 2941 (2010)

21. Du, H., Gong, M., Liu, R., Jiao, L. : Adaptive chaos clonal evolutionary programming algorithm. Science in China, Series F: Information Sciences. 48(5),579-595 (2005)

22. Zuo, X.Q., Fan,Y.S.: Chaotic-search-based immune algorithm for function optimization. Kongzhi Lilun Yu Yinyong/Control Theory and Applications. 23(6), 957960+966 (2006)
23. Zuo, X.Q., Li,S.Y. : The chaos artificial immune algorithm and its application to RBF neuro-fuzzy controller design. Proceedings of the IEEE International Conference on Systems, Man and Cybernetics. 3,2809-2814 (2003)

24. Alatas, B. : Chaotic bee colony algorithms for global numerical optimization. Expert Systems with Applications. 37(8),5682-5687 (2010)

25. Mingjun, J., Huanwen, T.: Application of chaos in simulated annealing. Chaos, Soitons and Fractals. 21(4),933941 (2004)

26. Hénon, M.: A two dimensional mapping with a strange attractor. Commun. Math.P hys. 50, 69-77 (1976).

27. Lozi, R.: Un attracteur etrange? du type attracteur de Hénon. J hys. 39(C5), 9-10 (1978)

28. Sprott, J.C. : chaos and Times-Series Analysis. (Oxford University Press,Oxford,UK). (2003)

29. Hunt, B.R.,Kennedy, J.A., Li, T.Y., Nusse,H.: The Theory of Chaotic Attractorss. Springer, New-York (2003)

30. Zeraoulia, E. Sprott, J.C.: A two-dimensional discrete mapping with $C^{\infty}$-multifold chaotic attractors. Electronic journal of theorical physics. 5(17), 107-120, (2008)

31. Aziz-Alaoui, A., Robert, C., Grebogi, C.: Dynamics of a Hénon-Lozi map, Chaos, Solitions Fractals, 12(11),2323-2341 (2001)

32. Misiurewicz, M.: strange attractors for the lozi mappings. annals of the new york academy of sciences, nonlinear dyn. 357, 348-358 (1980)

33. Sakurai, A.: orbit shifted shadowing property of generalized lozi maps. taiwanese journal of mathematics 14, (4), 1609-1621 (2010)

34. Coelho,L.D.S. : Tuning of PID Controller for an Automatic Regulator Voltage System using Chaotic Optimization Approach. Chaos, Solitons and Fractals. 39(4), 1504-1514 (2009)

35. Hamaizia, T., Lozi, R.:Improving Chaotic Optimization Algorithm using a new global locally averaged strategy. Proceedings ENPACS. (2011)

36. Shayeghi, H., Jalilzadeh, S., Shayanfar, H.A., Safari, A. : Robust PSS Design using Chaotic Optimization Algorithm for a Multimachine Power System. ECTI-CON 2009, Pattaya, Thailand. 40-43 (2009)

37. Shayeghi, H., Shayanfar, H.A., Jalilzade, S., Safari,A.: A PSO based Unified Power Flow Controller for Damping of Power System Oscillations. Energy Conversion and Management. 50(10), 2583-2592 (2009)

38. Cong, S., Li, G., Feng, X.: An Improved Algorithm of Chaos Optimization. Proceedings 8th IEEE International Conference on Control and Automation (2010) 